Mots. Les langages du politique

\title{
La parole en mouvement du patronat. CNPF-Medef
} 1981-2010

The forward movement of French employers' discourse. CNPF-Medef 1981-2010

La palabra en movimiento de la patronal. CNPF-Medef 1981-2010

Josette Lefèvre

\section{(2) OpenEdition}

Journals

Édition électronique

URL : https://journals.openedition.org/mots/21499

DOI : $10.4000 /$ mots. 21499

ISSN : 1960-6001

Éditeur

ENS Éditions

Édition imprimée

Date de publication : 16 décembre 2013

Pagination : 89-109

ISBN : 978-2-84788-527-9

ISSN : 0243-6450

\section{Référence électronique}

Josette Lefèvre, «La parole en mouvement du patronat. CNPF-Medef 1981-2010 », Mots. Les langages

du politique [En ligne], 103 | 2013, mis en ligne le 16 décembre 2015, consulté le 22 avril 2022. URL

http://journals.openedition.org/mots/21499; DOI : https://doi.org/10.4000/mots.21499

\section{(C) ENS Éditions}




\section{La parole en mouvement du patronat. CNPF-Medef 1981-2010}

Cette étude statistique de vocabulaire sur un corpus constitué de discours et de textes d'assemblées générales du CNPF et du Medef entre 1981 et $2010^{1}$ a pour objectif de répertorier le vocabulaire patronal sur une période assez longue pour saisir les caractères propres à cet univers lexical et, au-delà, d'appréhender le message diffusé. Comme pour le travail de recherche sur le discours syndical ouvrier de congrès ${ }^{2}$, nous avons choisi de nous focaliser sur le discours du sommet, celui produit pour les assemblées annuelles, qui distribue, contrôle et orchestre les usages lexicaux en interne pour les militants et adhérents de l'organisation mais joue et influe aussi en externe sur le gouvernement, les syndicats, les médias, l'opinion publique, etc. C'est donc la parole patronale officielle, parole collective engageant l'organisation entière, qui est l'objet de cette étude lexicométrique. La lexicométrie3 est une méthode au service de l'analyse de discours qui permet, au stade de l'interprétation des résultats, d'articuler deux approches complémentaires : d'une part, une approche quantitative basée sur un corpus clos, stratifié, à variables réduites pour l'exhaustivité des relevés et les calculs statistiques; d'autre part, une approche qualitative reposant sur l'examen des situations contextuelles. La démarche vise à appréhender, à travers le repérage des mots dits ou des mots tus, des alliances de mots, des lexicalisations et réseaux statistiques, des emprunts ou des inventivités, les stratégies qui traversent le discours.

Parmi les mots qui constituent cette parole officielle, le choix d'une dénomination revêt une dimension argumentative. Le nom que se donne un groupe participe tout autant à la constitution de son identité qu'à la construction de son positionnement dans l'espace social. Ainsi, c'est sous l'appellation

1. La constitution de ce corpus de textes patronaux a été réalisée avec l'aide financière de la DARES dans le cadre du projet de recherche du ministère du Travail sur les «Organisations d'employeurs en France» (2009-2011). Voir Amossé et al., 2012.

2. Voir Béroud, Lefèvre, 2010.

3. Pour cette étude, nous avons utilisé le logiciel Lexicoz réalisé sous la direction d’André Salem (Syled, Sorbonne nouvelle, Paris 3), notamment le programme des spécificités et les concordances pour la systématisation des analyses contextuelles. 
Confédération générale de la production française (CGPF) que se crée, en 1919, la première organisation patronale confédérale (Latournerie, 1996), pendant de la toute jeune Confédération générale du travail (CGT) constituée en 1895 par la fusion des syndicats ouvriers et des Bourses du travail. Aux mains des dirigeants de l'industrie lourde et des grandes entreprises, la CGPF est loin de regrouper l'ensemble des chefs d'entreprise. Aussi, la signature des accords de Matignon entre la CGPF et la CGT, en juin 1936, soulève-t-elle un vent de contestation des «petits et moyens patrons». La nécessité de souder le groupe pour conforter l'organisation va s'afficher dans une nouvelle dénomination, Confédération générale du patronat français :

Dans ces efforts, la terminologie eut un rôle primordial : la Confédération générale de la production française, conçue par beaucoup comme l'instrument des grands producteurs, devait se donner un nouveau nom où pouvaient se reconnaître d'autres catégories de chefs d'entreprise [...]. Dans le nouveau discours d'unité, présenté comme un discours spécifiquement patronal, les chefs d'entreprise n'étaient plus des industriels, des commerçants, des artisans, des métallurgistes, des détaillants, ils n'étaient plus petits, grands, ou moyens : ils étaient tous, avec ou sans salariés, des patrons, et formaient ensemble une nouvelle communauté dans la société : le patronat. (Kolboom, 1984)

L’organisation patronale rétablie après la Libération mise, par la transformation de son sigle et de son nom en Conseil national du patronat français (CNPF), sur sa nécessaire revalorisation patriotique (Conseil national) dans le cadre du programme politique de reconstruction du pays. Un peu plus de cinquante ans plus tard, après avoir surmonté les épreuves déstabilisatrices de mai 1968 et de mai 1981, et traversé plusieurs crises économiques et sociales, l'organisation patronale proclame sa refondation dans le Medef, Mouvement des entreprises de France, nom en décalage avec les appellations antérieures, reflet d'une ambition dévoilée :

Genèse d'un nom : «Mouvement des entreprises de France » a été retenu pour être notre nouveau nom car nous aimons le mot de «mouvement» qui correspond à notre slogan «En avant l'entreprise !» [...] «Des entreprises» parce que nous avons jugé que le terme de «patronat» était un peu démodé, ancien et que les entreprises sont naturellement ce que nous représentons. Enfin «de France» parce qu'il a été jugé nécessaire d'avoir cette accroche nationale, au moment où nous rentrons dans l'Europe, pour rappeler que notre collectivité doit réussir ensemble. (ErnestAntoine Seillière, Assemblée générale, 1998)

Ces transformations successives témoignent à la fois d'une stratégie d'adéquation aux temporalités sociopolitiques et d'un souci langagier, comme l'annonce Jean Gandois à l’Assemblée générale de 1995 : «Animer notre représentation sur l'ensemble du territoire : c'est mobiliser mais aussi donner à ceux qui parlent et agissent au nom du patronat une doctrine et un langage communs. » 
Un premier déchiffrage lexicométrique des textes patronaux met en évidence son système de répétition ainsi que les glissements lexicaux traduisant les évolutions liées au passage du CNPF au Medef. Dans un deuxième temps, quelques formes et lexies occupant une place centrale dans le corpus sont analysées.

\section{Analyse lexicométrique du corpus}

Le corpus, échantillon représentatif de la parole patronale officielle, est riche de 381139 occurrences réparties sur 17686 formes.

Formant l'ossature du discours, les formes et lexies majeures par la fréquence et la régularité d'emploi requièrent d'abord notre attention. Les occurrences cumulées des possessifs nous, notre et nos s'élèvent à 6218 et celles d'entreprise(s) à 4039 , ce qui propulse le lemme nous au $10^{\mathrm{e}}$ rang de l'index des fréquences à la suite des formes de, la, l', et, les, des, à, le, $d^{\prime}$, et le terme entreprise au $12^{\mathrm{e}}$ rang derrière en et devant la vingtaine de mots grammaticaux qui occupent le haut de l'index hiérarchique 4 . Nous et entreprise(s) tiennent ainsi une place singulière dans ce discours, l'articulent, en sont la grammaire. Pour s'assurer de ce rôle fonctionnel dans l'organisation textuelle, nous avons appliqué le programme des spécificités au corpus divisé en 26 parties-années.

Les 26 index qui en sont ressortis nous ont étonnée : il est en effet remarquable que sur une aussi longue période ( 30 ans) et sur un aussi grand nombre de textes de longueur, de nature et surtout de locuteurs différents, les mêmes termes se retrouvent parmi les dix premières formes lexicales de chacun des index de fréquences. Leur emploi et leur ventilation sont par conséquent d'une grande régularité dans l'ensemble du corpus. En tête de tous les index de fréquences, nous 5 et entreprise sont bien le pivot du dispositif discursif, en sont l'étendard.

Ces index font voir un deuxième sous-ensemble de formes un peu moins fréquentes avec des positions certes fluctuantes selon les années, mais qui perdurent. L'étude des résultats de l'analyse des spécificités par année, tout en donnant à voir les formes d'usage plus conjoncturel, confirme ces oscillations. La partition par décennie ${ }^{6}$, également testée, présente le double inconvénient d'écraser les moments clés des suremplois sans permettre la révélation des évolutions majeures du corpus. Les résultats de ces différentes partitions, par

4. L'index hiérarchique liste et ordonne toutes les formes du corpus : formes lexicales et formes grammaticales. Ces dernières (de, la, l', et, les, des, etc.), occupent naturellement les premières places.

5. Pour bien prendre la mesure de ce phénomène lexical, il est intéressant de comparer les corpus patronal et syndicaux : ainsi, dans le corpus patronal, la forme nous se situe au $16^{\mathrm{e}}$ rang de l'index lexicométrique, alors qu'elle n'occupe que le $99^{e}$ rang dans le corpus syndical, le $82^{\mathrm{e}}$ rang dans le corpus CGT et le $71^{\mathrm{e}}$ rang dans le corpus CFDT, confédération syndicale qui l'emploie le plus.

6. Les cinq premières formes lexicales par décennie sont : nous, entreprises, notre, entreprise, nos (partie 1981-1990); nous, entreprises, travail, entreprise, notre (partie 1991-2000); nous, entreprises, France, notre, pays (partie 2001-2010). 
années ou par décennies, ne révélant aucun clivage significatif, et estimant que sur ce corpus de 381139 occurrences le quantitatif faisait sens, nous avons finalement opté pour une analyse selon la partition CNPF/Medef, soit, d'une part, les textes des années 1981-1997 (187596 occurrences) et, d'autre part, ceux des années 1998-2010 (193543 occurrences), pour, dans une première approche de ce corpus inédit, visualiser nettement les grands événements lexicaux.

\section{Hautes fréquences et formes spécifiques}

Pour commencer, nous avons examiné la variation des positions des quinze premières formes lexicales du corpus dans les index CNPF et Medef (voir tableau 1 infra). Sur ces 15 premières formes du corpus Corpatot, 10 figurent dans les deux sous-parties CNPF et Medef: nous et entreprises confirment bien leur prééminence. Les formes notre, entreprise, nos, travail, pays, emploi, on, sociale, tout en n'occupant pas toujours le même rang - par exemple, pays glisse de la quatorzième à la sixième place -, demeurent installées dans les hautes fréquences. Davantage qu'un symbole, la sous-partie Medef enregistre la propulsion de France devant entreprise, et les montées explicites de salariés, croissance et État. Ces toutes premières observations tendent à établir la stabilité d'une part importante du vocabulaire majeur. En effet, seules temps (en suremploi CNPF), croissance et État (en suremploi Medef) ne se retrouvent pas parmi les quinze premières formes de Corpatot. Le phénomène le plus remarquable est l'entrée dans la liste, en septième position, de France, forme dont l'emploi dans Medef explose, en même temps que l'usage de pays progresse. Moins spectaculaire mais tout aussi significative est la montée de salariés.

\begin{tabular}{lll}
\hline $\begin{array}{l}\text { CNPF } \\
\text { (sous-corpus 1981-1997) }\end{array}$ & $\begin{array}{l}\text { Medef } \\
\text { (sous-corpus 1998-2010) }\end{array}$ & $\begin{array}{l}\text { Corpatot } \\
\text { (ensemble du corpus) }\end{array}$ \\
\hline nous & nous & nous \\
entreprises & entreprises & entreprises \\
notre & France & notre \\
entreprise & entreprise & nos \\
nos & notre & travail \\
travail & pays & France \\
CNPF & travail & pays \\
politique & nos & emploi \\
formation & emploi & on \\
emploi & on & sociale \\
on & sociale & formation \\
économique & Medef & politique \\
sociale & salariés & salariés \\
pays & croissance & économique \\
temps & État & \\
\hline
\end{tabular}

Tableau 1. Les 15 premières formes lexicales 


\section{Profil d'emploi des formes lexicales les plus fréquentes7}

Le tableau ci-dessous ordonne les 60 formes lexicales de fréquence (F) supérieure à 245 occurrences selon leur profil d'emploi : les suremplois de la souspartie CNPF dans la colonne de gauche, ceux de la sous-partie Medef dans la colonne de droite, les formes d'emploi équilibré (formes dites de base) ou peu clivantes (indice de spécificité inférieur à + 06) dans la colonne centrale.

\begin{tabular}{|c|c|c|c|c|c|}
\hline Suremplois CNPF & & $\begin{array}{l}\text { Emplois médians } \\
\text { et de base }\end{array}$ & & Suremplois Medef & \\
\hline entreprises & $F=2607$ & entreprise & $F=1432$ & France & $F=1019$ \\
\hline formation & $F=609$ & travail & $F=1096$ & pays & $F=819$ \\
\hline politique & $F=577$ & emploi & $F=695$ & salariés & $F=570$ \\
\hline CNPF & $F=537$ & sociale & $F=650$ & État & $F=550$ \\
\hline commission & $F=359$ & économique & $F=552$ & croissance & $F=542$ \\
\hline action & $F=349$ & temps & $F=496$ & développement & $F=443$ \\
\hline milliards & $F=286$ & aujourd'hui & $F=442$ & Medef & $F=365$ \\
\hline président & $F=284$ & ans & $F=406$ & publics & $F=355$ \\
\hline chefs d'entreprise & $F=276$ & taux & $F=383$ & heures & $F=341$ \\
\hline situation & $F=270$ & économie & $F=377$ & Europe & $F=328$ \\
\hline progrès & $F=256$ & année & $F=375$ & réforme & $F=304$ \\
\hline \multirow[t]{4}{*}{ organisation } & $F=249$ & niveau & $F=358$ & dialogue & $F=280$ \\
\hline & & années & $F=357$ & recherche & $F=263$ \\
\hline & & social & $F=355$ & entrepreneurs & $F=260$ \\
\hline & & compétitivité & $F=353$ & & \\
\hline
\end{tabular}

Tableau 2. Formes lexicales les plus fréquentes selon leur profil d'emploi

La colonne centrale des emplois fréquents les plus réguliers continue avec : place $(\mathrm{F}=346)$, sociaux $(\mathrm{F}=340)$, système $(\mathrm{F}=335)$, monde $(\mathrm{F}=329)$, professionnelle $(F=327)$, chômage $(F=319)$, partenaires $(F=303)$, marché $(F=303)$, ensemble $(F=295)$, nouvelles $(F=293)$, emplois $(F=292)$, loi $(F=284)$, nombre $(F=273)$, dépenses $(\mathrm{F}=268)$, jeunes $(\mathrm{F}=256)$, accord $(\mathrm{F}=23)$, charges $(\mathrm{F}=252)$, protection $(\mathrm{F}=251)$, gestion $(\mathrm{F}=245)$.

Ainsi, 34 des 60 formes les plus fréquentes du corpus se trouvent bien réparties, 20 (en italique) étant même de base. Ce qui permet de supposer l'existence

7. Le programme des spécificités permet d'évaluer la sous-fréquence (ventilation de la fréquence totale) de chaque forme pour chaque partie par rapport à sa fréquence totale dans le corpus. Ainsi chaque forme retenue est étiquetée d'emploi "spécifique» ou d'emploi «banal». «Spécifique positif» c'est-à-dire suremployée dans telle partie, "spécifique négatif » c'est-àdire sous-employée dans telle autre partie, «banal » c'est-à-dire au-dessus du seuil à partir duquel une probabilité devient pertinente. Car plus une probabilité est petite, plus l'événement saisi est statistiquement improbable et linguistiquement intéressant. Un coefficient de spécificité donne une idée de grandeur de cette probabilité : plus un indice est élevé, plus la probabilité est petite, plus la spécificité est significative. 
d'un important noyau lexical stabilisé, imperméable aux aléas conjoncturels. Parmi les 26 formes les plus fréquentes spécifiques, 12 se rangent du côté CNPF, 14 du côté Medef. Les formes à haut indice de spécificité (supérieur à + 09) sont en italique : 7 formes pour la partie CNPF, 9 formes pour la partie Medef. Côté CNPF, la liste rassemble des mots concrets liés à la vie organisationnelle et à son périmètre d'action; côté Medef, elle découvre des mots plus abstraits concernant le champ plus large de l'économie générale et politique. Une analyse des termes les plus spécifiques de chaque partie va préciser ces premiers constats.

\section{Les formes et lexies les plus spécifiques}

Dans la configuration binaire de cette démonstration, les spécificités positives de l'une faisant les spécificités négatives de l'autre, nous nous référons uniquement aux termes suremployés de chaque sous-partie. Et, pour nous en tenir aux clivages les plus marquants, nous ne retenons que les formes à indice supérieur à + 09. 33 formes et lexies répondent à ces critères ultra-sélectifs pour la partie CNPF, contre 37 pour la partie Medef.

\begin{tabular}{llll}
\hline CNPF & \multicolumn{3}{c}{ Medef } \\
\hline CNPF & +51 & Medef* & +51 \\
patronales & +39 & France & +51 \\
unions & +36 & entrepreneurs & +32 \\
patronale & +32 & euro & +27 \\
je & +28 & Union & +25 \\
unions patronales* & +27 & développement durable* & +23 \\
nous & +27 & 35 heures & +22 \\
nos & +22 & dialogue & +18 \\
action & +22 & oui & +18 \\
politique contractuelle* & +21 & pays & +18 \\
1992 & +20 & parce que & +17 \\
francs & +19 & soins & +16 \\
avons & +15 & Unioneuropéenne & +15 \\
progrès & +15 & réformer & +14 \\
commission & +15 & démocratie & +14 \\
management & +14 & réforme & +14 \\
politique & +14 & refondation sociale & +13 \\
entreprises & +13 & attractivité & +13 \\
crise & +13 & santé & +13 \\
chefs d'entreprise & +12 & accès & +13 \\
temps de travail & +12 & durable & +12 \\
professions & +12 & européenne & +12 \\
territoriale & +12 & français & +11 \\
TVA & +11 & développement & +11 \\
mesures & +11 & États-Unis & +11 \\
nous avons & +11 & offre & +11 \\
prix & +11 & Medefterritoriaux & +11 \\
intervention & +11 & zone & +11 \\
& & &
\end{tabular}




\begin{tabular}{llll} 
difficile & +11 & refondation & +11 \\
clubs & +11 & mondialisation & +11 \\
$U P^{\star}$ & +10 & proposons & +11 \\
réduction & +10 & heures & +11 \\
doctrine* & +10 & voisins & +10 \\
& & salarié & +10 \\
& innovation & +10 \\
& recherche & +10 \\
& gouvernance & +10 \\
& & croissance & +10 \\
\hline
\end{tabular}

Tableau 3. Plus forts suremplois selon la partition CNPF/Medef

Ce qui frappe dans la liste CNPF, c'est la présence, à la suite du fort suremploi de CNPF lié bien sûr au changement de sigle, d'un ensemble de formes - patronale(s), unions, action, commission, territoriale, progrès, management, politique, professions, clubs - servant principalement à la désignation des structures ou au fonctionnement de l'organisation patronale (Commission politique sociale), ou bien encore s'insérant dans le nom d'institutions proches: Association pour le progrès du management (APM), Commission Progrès des entreprises, Entreprise et Progrès... Sur les 489 occurrences cumulées des huit formes de la souche patron-, seulement 54 s'inscrivent dans la partie Medef, notamment après 2005. Patronales passe ainsi de 184 à 16 occurrences (cotisations patronales), patronale de 125 à 5, patronaux de 36 à 4, patronal de 30 à 6, patronat de 32 à 9 et patronats de 14 à 1 . Le quasi-abandon des mots de la famille «patron » après 1998 engendre un fléchissement d'usage voire la disparition de plusieurs lexies : unions patronales (UP), instances, organisations... patronales; union, organisation (OP), unité, cohésion, communauté, solidarité, doctrine, pensée, action... patronale, militantisme, corps, monde, succès, syndicalisme, exécutif, réseau... patronal, mandats, mandataires... patronaux, clubs et mouvement de pensée patronaux, etc.

L'intention de gommer du discours les formes et lexies étiquetées «patronat», de masquer l'appartenance à la «classe patronale» transparaît clairement. Le nom même de chefs d'entreprise, avec un profil d'emploi à la baisse (comme dirigeants) semble menacé par celui plus mythifié d'entrepreneurs. La lexie politique contractuelle 8 est remisée au profit de dialogue social, au sens plus flou.

Une autre série ressort, composée de formes moins fréquentes dont les emplois se concentrent sur des analyses plus conjoncturelles ou très ciblées. La référence aux temps forts de l'action patronale est très présente, notamment vis-à-vis du gouvernement (en fort suremploi), francs, prix («contrôle des prix», «liberté des prix»), intervention («intervention incessante de l’État»,

8. L'astérisque signale une forme propre à la sous-partie. 
«intervention constante des pouvoirs publics»), TVA9, réduction (« réduction du temps de travail », "réduction des dépenses publiques», "réduction des dépenses de fonctionnement de l'État»), temps de travail, mesures. L'usage du substantif politique renvoyant à l'action de l'État (politique sociale, économique, salariale...) est de manière générale plus important à cette époque, comme celui de mesures (mesures gouvernementales).

92 des 112 emplois totaux du mot crise le sont dans cette partie : le poids de la crise, les effets de la crise, pour sortir de la crise, la crise internationale, la crise française, la crise monétaire, la crise financière. Son environnement contextuel découvre un usage très politique. La rhétorique autour de crise participe, le plus souvent, à la disqualification de l'intervention de l'État dans le champ de l'économie : "Sans libertés, notre économie restera anémiée, incapable de sortir de la crise française et de rejoindre les nations qui renouent avec la croissance. Sans libertés, nous resterons une économie administrée et demain dépassée. » (AG 1984) Les argumentations développées par le CNPF sur cette thématique font d'autant plus ressortir l'absence d'une véritable analyse des causes de la crise de $2008^{10}$.

Dès 1986, l'organisation patronale, qui a toujours soutenu la construction européenne, développe une stratégie d'action offensive en vue d' "assurer la libéralisation de l'économie pour réussir l'entrée en vigueur de l'acte unique européen », ce qu'explique la fréquence élevée de la forme 1992. Une activité patronale importante est mobilisée pour se saisir de cette opportunité européenne, comme le souligne l'extrait suivant :

Cette Europe doit être l'occasion de mettre en pratique une dérégulation de l'économie, à l'image de ce que nous essayons de faire en France, comme dans d'autres pays européens. Elle ne doit pas être le refuge de la bureaucratie et la revanche d'une administration frustrée par le recul de l'État. L'Europe doit être cet espace de liberté économique sans lequel nos entreprises ne peuvent pas donner le meilleur d'elles-mêmes.

L'autre écueil se situe sur le plan social : le risque majeur pour l'Europe des entreprises serait de les voir s'aligner dans un nouvel espace social européen qui aurait pour effet, là encore, de rétablir tout ce que nous avons réussi à supprimer. Je pense notamment à cette tentation, qui viendra tout naturellement, d'essayer d'harmoniser les conditions de travail dans l'Europe. Cela représenterait une illusion et un danger. Vous savez combien je tiens à la décentralisation du dialogue

9. La hausse de la TVA en période de blocage des prix suscite une vive réaction patronale.

10. «Nous nous devons deuxièmement de dire que la solution à la crise existe. Elle passe par l'entreprise, par les entreprises, et par l'esprit d'entreprise. Et c'est pour cela que notre rassemblement aujourd'hui est si important. C'est pour dire que la solution existe, qu'elle passe par l'entreprise et l'entreprise c'est nous tous! Ne nous laissons pas abuser par le schéma simpliste selon lequel il faudrait adopter soit une politique de relance soit une politique d'austérité car la gestion rigoureuse comme l'ambition de croissance sont également nécessaires et souhaitables. Le choix devant lequel notre pays se trouve est tout autre : non pas relance ou austérité, mais compétitivité ou marasme. » (AG 2010) 
social et à l'institution de conditions de travail adaptées aux possibilités des entreprises. Dans l'édification de l'Europe, ne tombons pas dans le piège d'un espace social européen uniforme. (AG 1987)

Aucun autre mot-clé du lexique patronal ne figure dans la liste complète des spécificités en positif de cette partie, ce qui tend à prouver l'absence de mouvement lexical significatif d'une évolution de la doctrine. Le «parti de l'entreprise », délesté de son caractère dynastique, peut désormais, au nom de l'intérêt général, envisager d'insuffler l'«esprit d'entreprise » au corps social tout entier.

C'est donc l'ensemble de la terminologie perçue comme trop identitaire de l'univers patronal, marqueur des antagonismes de classe dans la société, obstacle idéologique à l'avènement de l'ère entrepreneuriale, qui s'efface avec le changement de nom.

Outre les termes qui suivent la transformation de CNPF en Medef, comme refondation sociale, Medef territoriaux, ce qui caractérise le plus la partie Medef, c'est l'importance exceptionnelle qu'y prend le terme France (+ 51), devançant même en nombre d'occurrences entreprise, avec la forte progression d'Union européenne et de pays. La consécration du mot entrepreneurs (+32) est bien nette : attesté dès 1981, le mot voit ses emplois progresser à partir de $1995^{11}$. En 2001, Ernest-Antoine Seillière fabrique du mythe entrepreneurial avec cette définition dithyrambique :

Qu'est ce qu'un entrepreneur? C'est une femme ou un homme, je le dis souvent, qui se met à risques pour créer les biens et les services dans notre pays, qui anticipe, qui projette, qui avance, qui ose, qui innove, qui embauche, qui investit, qui forme, qui décide, qui mène les hommes, qui dialogue, qui prend des responsabilités, envers ses clients, ses salariés, ses actionnaires, qui les assume toujours. II accepte les lois du marché, il respecte les lois de la République, il domine la concurrence, il recherche la compétition. Il est loyal, il cherche le succès, il aime la réussite, il rend des comptes, il accepte l'échec, il recommence. L'entrepreneur a le moral et la pêche! Il a un beau métier. Il en est fier !

Le libéralisme participatif ouvre le large éventail des opportunités de «codécision » qui peuvent s'établir entre salarié(s) et employeur(s). Partenaires dans l'entreprise avec les actionnaires et les clients, les salariés, qui ne représentent plus la classe des travailleurs ${ }^{12}$, font corps avec l'équipe entrepreneuriale; à ce titre, ils doivent être informés des contraintes qui pèsent sur leur entreprise. Diverses formules fleurissent pourvaloriser les processus d'individualisation

\footnotetext{
11. Le terme entreprenants testé en 1984, «L'année des entreprenants », n’a pas eu de suite. «[...] agriculteurs, artisans, professions libérales et chefs d'entreprise, ceux que nous appelons les “entreprenants" [...]. Ces “entreprenants”, c'est-à-dire ceux qui risquent et entreprennent, ceux pour qui l'échec professionnel est souvent synonyme de faillite personnelle... » (AG 1985)

12. Lefèvre, 1993 et 2003.
} 
et de responsabilisation du salarié : «permettre à chaque salarié de devenir acteur de son projet professionnel», "faire de chaque salarié un acteur de son développement », « développer l'actionnariat salarié »; ainsi, « une fois actionnaires, les collaborateurs travaillent et agissent à la fois comme salariés pour l'emploi et comme actionnaires pour le capital!»

L'astérisque accolé à démocratie signale un mot propre à la partie, et donc son absence dans la partie CNPF, constat qui interroge dans les deux configurations sur la référence à la démocratie. La plupart des 47 occurrences renvoient à la démocratie sociale à construire (la lexie démocratie sociale est attestée 25 fois). Dans quelques autres usages, c'est un dessein plus large du rôle des «producteurs » dans la société qui est proclamé, ou c’est le lien entre démocratie politique et économie de marché qui est construit pour argumenter face à la menace de protectionnisme.

Sur un total de 1019 occurrences, France est attesté 777 fois. D’autres formes suremployées renforcent encore la montée en puissance de cette thématique : français et Français (l'adjectif féminin est d'emploi banal), notre pays, dans ce pays : « Notre seule tâche est en fait de prouver que ce qui est bon pour l'entreprise est bon pour la France », déclare Denis Kessler à Strasbourg en 1998. Le paradigme France, placé au même niveau qu'entreprise comme dans le slogan «En avant l'entreprise! En avant la France ! », envahit l'ensemble du discours, y tissant des liens étroits entre l'économique, le social et le politique. Cette analogie avec l'entreprise légitime les nouveaux modes de gouvernance qui, au nom de la mondialisation de l'économie, doivent être mis en place. Le moindre usage des lexies entreprises françaises et nos entreprises est révélateur de ce changement d'échelle. L'ensemble des mots qui couvre ce mouvement, Union européenne, Europe, zone euro, développement, développement durable, mondialisation, États-Unis, voisins, innovation, croissance, recherche, attractivité, gouvernance, dialogue, dialogue social, dialogue économique, société civile, démocratie, démocratie sociale est symptomatique du poids de cet élargissement discursif. L'Entreprise France ne peut prospérer que dans un État géré comme une entreprise : «moins d’État, mieux d’État».

Les thématiques ${ }^{13}$ du «retard français» dans la «compétition européenne et mondiale » et de la nécessaire « réforme de l’État» avec « un nouveau partage des responsabilités entre État et partenaires sociaux» pour «aller de la société d'assistance à la société de travail », en passant par les réformes du «système de protection sociale», les «nouveaux domaines d'action concernant la formation et l'éducation » s'enchevêtrent dans un maillage de plus en plus serré. Sous la formulation «l'esprit d'entreprise c'est aujourd'hui l'esprit de réforme », c'est un «vrai projet global de société pour la France et les Français» que le Medef s'em-

13. Les thématiques ne sont certes pas nouvelles mais amplifiées. Ernest-Antoine Seillière qualifie le «modèle français » d' " archaïque » dans son discours de clôture de l'AG 1997. 
ploie à faire advenir. II revendique une place à part entière dans la démocratie parce que «nous sommes une partie essentielle de la société civile», et le dialogue économique pour «un partenariat avec ceux qui nous gouvernent». Dans ce programme, le politique et le social doivent servir de base arrière à l'économique. Cette socialisation dans l'intérêt de la sphère économique privée se doit d'être non seulement d'excellence mais encore compétitive.

Les phrases s'enchaînent pour dire ce dont «la France a besoin » pour «tenir son rang en Europe et dans le monde», déplorer les «charges sociales qui pèsent très lourdement en France », "les coûts de production actuels en France », constater que «l'image économique de la France est floue», inciter à «mettre la France sur la voie de l'excellence», renforcer «l'attractivité du territoire français », «l'attractivité du site économique français ». Les compétences françaises sont évaluées par rapport à la moyenne européenne, aux autres pays européens, aux pays voisins, aux pays émergents. C'est l'image d'une France dont l'identité est réduite à n'être qu'un territoire à la traîne dans la compétition globale, qui est dépeinte. L'«urgence d'une gouvernance économique européenne » étaie le raisonnement sur l'obligation de réforme (réformes est de base). Il faut réformer au nom de l'Europe :

Ensuite, deuxième raison d'être optimiste : les hommes politiques perdent de plus en plus de pouvoir. C'est le cas sur le plan économique, sur le plan monétaire grâce à l'ouverture des frontières, à l'Europe, à Bruxelles, grâce à l'euro dont on ne dira jamais assez combien il a permis de dépolitiser, ou presque, le sujet de la monnaie et combien il a mis la France à l'abri de décisions hasardeuses telles qu'on les prenait dans le passé. Donc, les hommes politiques perdent une partie de leur pouvoir économique. Merci l’Europe! (AG, 2000)

L'argument démographique, liant croissance économique et croissance démographique, est développé par comparaison quantitative et qualitative des populations entre elles: «Asie plus jeune, avec une main-d'œuvre de plus en plus qualifiée». Le vieillissement de la population européenne («perte de dynamisme», «vieillissement de l’Europe», «une Europe vieillie», « déclin démographique en Europe») «dans un monde en expansion» (États-Unis, Chine, Inde) est une thématique qui s'exprime sur un ton fataliste et alarmiste. La ritournelle sur la non-adéquation des populations aux besoins du marché du travail ou sur la problématique de «la compétitivité et l'attractivité du site de production France» renforce encore le côté anxiogène du propos :

Prévenir à temps les graves conséquences du vieillissement de notre population [...]. Oui, cette contraction du nombre potentiel d'actifs et cette dilatation du nombre de retraités est gravement préoccupante, particulièrement si l'on considère que de nombreux pays au monde connaissent une expansion démographique. Moins de producteurs sur notre "site de production», moins de consommateurs sur notre «site de consommation », il faut agir en dépit de ce renversement démographique. (AG 2000) 
Le dossier des 35 heures, «le Big Bordel des 35 heures», devenu «le ferment d'une unité patronale sans précédent », a sans aucun doute facilité une transformation de l'organisation à l'étude depuis 1974, prête pour le cinquantenaire du CNPF14 avec pour nom Conseil des entreprises françaises, mais le pas n'a pu alors être franchi. Plus d'une dizaine d'années après que le mot travailleurs a disparu du vocabulaire syndical, l'estompage du mot patronat s'effectue à son tour. Les profils d'emploi du radical «patron-» dans les textes CFDT et CGT s’orientent à la baisse, à partir de 1992 à la CFDT et 1995 à la CGT. La fin de la lutte de classes sonne avec le nouveau lexique de partenariat social. L'objectif patronal de citoyenneté entrepreneuriale, c'est-à-dire «l'engagement de l'entreprise dans la cité», va pouvoir se décliner dans les énoncés.

Les syntagmes verbaux spécifiques de chacune des parties sont révélateurs de cette progression : le nous avons CNPF donne à voir une institution essentiellement corporatiste rendant d'abord compte d'une action orientée vers la défense de ses prérogatives pour exister en tant que groupe uni dans un environnement hostile, le discours à visée interne se fait didactique; quand le nous proposons et le parce que Medef laissent davantage voir un mouvement pouvant enfin œuvrer à faire advenir sa vision du monde, avec un discours à visée élargie agencé pour convaincre et produire du consensus tout en veillant à assurer sa cohésion idéologique interne. Ces univers lexicaux se prolongent et s'entrelacent depuis le milieu des années quatre-vingt avec la réhabilitation de l'entreprise et la dissémination du vocabulaire européen.

En à peine plus de deux ans, nous avons installé la parole de chefs d'entreprise dans tous les débats publics. Nous avons remis des mots à la mode, lancé des slogans et forgé des concepts. Tous ont acquis la force de l'évidence. Rappelez-vous le premier d'entre eux : réconcilier les Français avec l'entreprise. N'ayez aucun doute, cette réconciliation est en marche! (Laurence Parisot, AG 2008)

\section{Hautes fréquences et formes à profil plat}

La mise en lumière de ces formes plus ou moins chahutées ne doit pas se faire au détriment de celles qui sont mieux réparties dans le texte (voir tableau 4 ciaprès). Elles sont le socle lexical stabilisé et répété sur lequel repose le discours patronal, son patrimoine lexical.

14. «Nous devons adapter nos structures pour être un acteur influent. J'espère que les manifestations de notre cinquantenaire, dont le comité de pilotage est présidé par ErnestAntoine Seillière, permettront à la réforme de l'institution sur laquelle Victor Scherrer travaille, de prendre corps. Ce cinquantenaire, nous le célébrerons au Futuroscope à Poitiers, le 17 septembre 1996.» (AG 1995) 


\begin{tabular}{|c|c|c|c|c|}
\hline entreprise & protection & plan & possible & réseau \\
\hline travail & gestion & augmentation & investissement & général \\
\hline tous & durée & baisse & besoins & effort \\
\hline économique & liberté & grands & résultats & équilibre \\
\hline aujourd'hui & activité & efficacité & grande & impôt \\
\hline économie & toute & responsabilité & qualité & nécessité \\
\hline social & nouveau & objectif & salaire & national \\
\hline compétitivité & cotisations & coûts & épargne & réformes \\
\hline place & environnement & terrain & prélèvements & service \\
\hline sociaux & conditions & responsabilités & objectifs & capacité \\
\hline professionnelle & propositions & marchés & productivité & priorité \\
\hline marché & moyens & règles & sens & déficit \\
\hline ensemble & négociation & accords & solidarité & hommes \\
\hline toutes & française & intérêt & renforcer & relations \\
\hline emplois & politiques & nationale & volonté & syndicats \\
\hline dépenses & coût & salaires & contraintes & efforts \\
\hline jeunes & projet & véritable & améliorer & succès \\
\hline accord & évolution & besoin & industrie & réalité \\
\hline charges & concurrence & nécessaire & confiance & taxe \\
\hline
\end{tabular}

Tableau 4. Premières formes de base dans l'ordre fréquentiel

Un premier ensemble de formes relève du vocabulaire socio-économique général : entreprise, travail, économique, économie, social, emplois, accord, activité, nouveau, cotisations, négociation, etc. Un deuxième ensemble met en évidence le lexique du registre économique libéral : compétitivité («Lutter pour la compétitivité, c'est aussi développer le consensus social à l'intérieur des entreprises »; AG 1984), marché (marché du travail, lois du marché, parts de marché), dépenses (dépenses sociales, publiques, de santé, de protection sociale, d'assurance maladie), charges (poids des charges sociales, collectives, salariales, publiques, sur le travail, baisse des charges pour accroître l'embauche et le pouvoir d'achat), gestion, liberté ("La liberté tout court, c'est, pour le CNPF, celle qui conduit à la déréglementation »; ibid.), coût(s) (coût du travail, coût du SMIC, réduire les coûts), concurrence (concurrence étrangère, des pays émergents, du Sud, des pays neufs, mondiale), efficacité, investissement, épargne, prélèvements (prélèvements obligatoires, fiscaux, sociaux), productivité (gains de productivité, efforts de productivité, productivité du travail), contraintes («Desserrer les contraintes en matière d'emploi, ce n'est pas accroître la précarité, c'est permettre aux entrepreneurs d'exprimer pleinement leur goût d'entreprendre et leurvolonté d'embaucher : c'est déverrouiller l'emploi »; AG 1997), etc. Un troisième ensemble regroupe des termes abstraits porteurs des valeurs développées par l'organisation : liberté, responsabilité(s), sens, solidarité, volonté, confiance, effort(s), réalité, etc. 
Parce que les valeurs de l'entreprise doivent être transmises aux jeunes : une culture d'innovation, le goût du risque, l'esprit d'initiative, le sens des responsabilités (les prendre et les assumer), le sens des opportunités, l'ouverture au monde et la curiosité d'esprit, l'autonomie mais aussi le goût du travail en équipe, la créativité, la vitalité... Bref, des valeurs et des comportements qui incitent les hommes à être entrepreneurs de leur propre vie, à être en veille et à agir, plutôt qu'à subir. (AG 2002)

\section{Éclairages contextuels}

\section{Nous, du parti de l'entreprise à l'entreprise France}

«Nous sommes le parti de l'entreprise. Notre rôle est de défendre la mission économique des entreprises contre tout ce qui la menace et de promouvoir les entreprises auprès de tous ceux qui les ignorent » : c'est par cette phrase qu'Yvon Gattaz, président du CNPF, ouvre l'Assemblée générale du CNPF en décembre 1981.

Locuteur collectif omniprésent, le cumul des occurrences de nous et des adjectifs possessifs notre et nos (6 218 occurrences au total) enserre le discours. L'examen des concordances indique qu'il s'agit dans la majorité des cas d'un nous collectif inclusif en position de sujet. Le référent est rarement apposé, il est le plus souvent incertain : nous, (les) chefs d'entreprise(s) ne se rencontre que 26 fois, nous (les) entrepreneurs (de France) 22 fois, nous Medef 9 fois, nous Français 2 fois, nous entreprises de France 2 fois. Cette ambiguïté référentielle produit un effet d'homogénéité que la nomination des diverses instances de l'organisation patronale troublerait. C'est un nous à géométrie variable qui peut rassembler l'organisation tout entière, les participants à l'Assemblée générale, les permanents, les militants, les adhérents, les entrepreneurs, les salariés et, au-delà, tous les Français qui peuvent (doivent) adhérer au projet patronal.

C'est à nous tous, entrepreneurs et salariés des entreprises de France que je m'adresse aujourd'hui. Et pas, pour une fois, principalement aux pouvoirs publics et à l'État. Car la réussite économique et sociale de la France, c'est nous tous qui l'accomplissons ensemble, et c'est pour cela que nous devons promouvoir ensemble, en acteurs et en partenaires de la société civile, un projet pour la réussite économique et sociale de notre pays. (AG 1998).

C'est l'ensemble de tous ces nous «indistincts »'15 qui donne sa force à l'Organisation et lui permet de présenter ses idées sous le mode universel. Un nous englobant qui contrôle la cohésion idéologique du groupe, que vient renfor-

15. La revue Mots a consacré un numéro spécial à l'usage du «nous » en politique (1985). 
cer encore l'usage tout aussi massif des possessifs notre et nos. La légitimité du nous est constamment réaffirmée par le rappel des choix communs antérieurs, d'où la répétition nécessaire des mots-valeurs partagés et les fréquents retours sur les activités passées:

Mais, tous ensemble, par notre cohésion et notre combat au coude à coude si bien symbolisés par notre rassemblement fraternel et massif de Villepinte en décembre 1982, nous avons sauvé l'essentiel : l'image de l'entreprise dans la conscience des Français et l'esprit d'entreprise. Croyez-moi, ce n'est pas rien dans les valeurs d'une société.

L'une des valeurs qui nous est la plus chère : l'entreprise privée, était jetée en pâture à l'opinion publique. Quatre ans après, tous ensemble, nous avons su retourner cette opinion à notre profit.

Beaucoup plus qu'une victoire du monde patronal, c'est un retournement historique de l'opinion publique tout entière. Mais la révolution culturelle que nous avons faite a eu une autre conséquence plus facile à mesurer. En prônant en permanence notre attachement à cette liberté d'entreprendre qui date de 1791, en lui donnant une définition concrète, nous avons été les précurseurs du moins d’État. En luttant contre l'interventionnisme étatique sous toutes ses formes (rappelez-vous : «moins de charges, mais aussi moins d'aides»), en montrant la méconnaissance totale de la réalité économique de nos gouvernants, en déstabilisant leurs idées reçues, nous avons, les premiers, contribué à l'éclosion des idées libérales. (AG 1985)

Les syntagmes verbaux les plus récurrents conduisent des énoncés normatifs et définitoires fermés à toute contradiction : nous avons rend compte de l'action passée ("nous avons développé quelques idées simples», "nous avons engagé la bataille de la flexibilité»), nous sommes énonce ce que le «nous» doit être («nous sommes par nature des conquérants », « nous sommes, nous les entrepreneurs, des démocrates et des républicains et nous ne sommes au service des ambitions politiques de personne, ni parti, ni leader», "nous sommes la France qui produit, la France qui emploie, la France qui investit, la France qui innove, la France qui risque, la France qui forme, la France qui veut, la France des jeunes, la France qui gagne », " nous sommes prêts à gérer avec les syndicats, dans le cadre d'un paritarisme renouvelé, ce grand système de retraite couvrant tous les salariés du secteur privé »). D’autres syntagmes assignent les objectifs à atteindre, l'action à venir : nous voulons (« nous voulons vraiment que les Medef territoriaux représentent l'entrepreneur, qu'ils influencent, qu'ils communiquent et qu'ils s'imposent dans leur circonscription »), nous devons ("nous devons assurer en quelque sorte un service après vente de nos idées»), nous allons («nous sommes une partie essentielle de la société civile et nous voulons faire en sorte que s'établisse ce partenariat entre ceux qui nous gouvernent et ceux qui produisent les biens et les services de notre pays. Nous allons nous faire entendre»), nous proposons ("tout ce que nous proposons sans relâche s'inscrit dans une Vision, s'enracine dans des Valeurs, suppose une Volonté »). Le recours aux verbes constatifs est plus 
limité : nous savons («nous savons qu'avec la mondialisation, l'euro et l'Internet, s'établir ailleurs, produire ailleurs, externaliser, délocaliser est une vraie tentation»), nous voyons («nous voyons poindre la nouvelle refondation sociale, celle qui ouvre sur l'accord d'entreprise, conclu au plus près du terrain, au plus près de la réalité, au plus près de la diversité, au plus près de l'intérêt de chaque salarié»).

C'est le pronom on aux 677 occurrences bien réparties aussi qui prend en charge les phrases non assumées par le pronom nous; cette distanciation du locuteur permet d'objectiver le propos : «on ne peut faire de l'emploi sans croissance », " on n'a jamais réussi dans la vie en travaillant moins». Par ailleurs, on endosse aussi le rôle de qui n'est pas nous ; la plupart du temps, cet «Autre » est l'État ou le gouvernement en place : «on multiplie les lois d'un autre âge ", " on laisse bourgeonner les réglementations », " on nous a écoutés poliment», «rappelons-nous que l'écotaxe dont on nous a longtemps menacé n'avait pas pour but la protection de l'environnement, mais le financement des 35 heures, véritable tonneau des Danaïdes».

L'environnement des possessifs notre et nos renseigne aussi sur la figure du nous portée par l'organisation. Les syntagmes nos entreprises, notre action, nos organisations, nos structures, suremployés avant 1998, donnent à voir un nous davantage centré sur l'espace économique et social de l'entreprise : nous signifie entreprise. Ceux en suremploi après 1998, notre pays, nos voisins, nos valeurs, attestent d'un nous élargi à l'espace national : nous signifie France. Il ne s'agit pas d'une opposition ; les deux nous s'imbriquent tout naturellement, comme l'illustre cette formule prononcée par Ernest-Antoine Seillière en clôture de l’Assemblée générale de décembre 1997 - «Vive les entrepreneurs et, puisqu'il s'agit à la fois de nous et d'elle, vive la France»-, mais plutôt d'une annexion destinée non pas à une mutation mais au renforcement du «nous» originel, c'est-à-dire du même groupe social : «Nous sommes le parti de l'entreprise », donc « Nous sommes les porte-parole de la France qui bouge, de la France qui en veut, de la France moderne, de la France ambitieuse, de la France conquérante!» (AG 2002)

\section{Les occurrences du terme entreprise}

Occupant une place centrale dans le discours, entreprise fonctionne le plus souvent en emploi absolu. Parfois un adjectif en précise l'origine - entreprises françaises $(F=200)$, nos entreprises $(F=150)$, entreprises allemandes $(F=16)$, européennes $(F=15)$, étrangères $(F=11)$, américaines $(F=8)-$, la taille : grandes $(F=32)$, petites $(F=30)$, moyennes $(F=15)$ entreprises ( Sur chacun des thèmes dont nous nous occupons, nous nous sommes efforcés d'intégrer les problèmes spécifiques des petites et moyennes entreprises. Il reste beaucoup à faire et nous poursuivons nos efforts en 1999 ") -, ou au contraire insiste 
sur leur communauté : toutes les entreprises $(F=49)$. Les entreprises $d u C A C$ 40 et les entreprises multinationales n'apparaissent que 5 fois en tout. Le sigle $P M E$, attesté 186 fois, surtout après 1998, occupe une place modeste dans le corpus, comparé aux 4039 occurrences du terme entreprise. Le Medef suggère la mise en place de "partenariats intelligents » entre les grands groupes et les PME. Les questions de création, d'aide et de développement des PME sont déléguées aux Unions territoriales.

Dans son environnement contextuel circulent la plupart des formes de base du vocabulaire économique : compétitivité, liberté, efficacité, charges, dépenses, investissement(s), taux d'intérêt(s), marché(s), coût(s), contraintes, environnement, succès, etc. Fil rouge du discours, c'est par son entremise que s'avance le corps de doctrine patronale marqué du martèlement des mêmes mots.

\section{Les occurrences du terme économique}

Cumulées, les occurrences d'économique (1116 au total pour l'adjectif et le substantif économie) et de social (1468 au total pour les flexions adjectivales) font partie des plus hautes fréquences émaillant le discours selon une répartition équilibrée. Se rangeant par leurs fréquences à la suite de nous et d'entreprise(s), elles consacrent le domaine de compétence et d'action de l'organisation : "Le CNPF n'a pas à s'assimiler ni à se substituer à aucun parti, quel qu'il soit. À chacun son métier : le nôtre est économique et social, et notre langage sera d'autant plus fort et plus crédible qu'il sera dépouillé de sousentendus.» (AG 1984)

Cinq lexies seulement dépassent toutefois les 20 occurrences : politique(s) économique(s), situation économique, développement économique, croissance économique, activité économique. La plupart des occurrences font corps avec l'évolution de la palette lexicale du registre économique, ventilées sur l'ensemble du corpus ou regroupées dans une des deux parties. L'ensemble concourt à la formation du dispositif répétitif servant d'argument à l'organisation patronale pour asseoir son projet global de société :

- principales lexies : compétitivité, compétition, débat, décideurs, domaine, dynamisme, efficacité, environnement, espace, évolution, histoire, impératifs, intérêts, mondialisation, partenaires, performance, poids, progrès, puissances, ralentissement, réalité, reprise, santé, réalités, réussite, stagnation, stratégie, tissu, vie... économique(s);

- lexies majoritairement CNPF : adaptation, avenir, bataille, conjoncture, crise, déflation, difficultés, force, guerre, hégémonie, incertitudes, licenciements, nécessités, philosophie, récession, redressement, responsabilités, riposte, système, survie... économique(s);

- lexies majoritairement Medef : acteurs, activités, attractivité, bien-être, contraintes, convergence, destin, dialogue, égalité des chances, enjeux, 
équilibre, essoufflement, gouvernance, initiative, jeu, licenciement, modèle, mutations, nouvelles forces, paysage, sur le plan, projet, régulations, rentabilité, stratégies, tempête, zone... économique(s).

Le réseau contextuel d'économie met en évidence deux secteurs antagoniques : l'économie administrée, assistée, dirigée, contrainte, mixte, d'hier, est opposée à l'économie de marché (lexie la plus fréquente), de liberté, de libre entreprise, de compétition (et de liberté), moderne (et compétitive), compétitive, concurrentielle, libérale, mondiale, mondialisée...

Le monde est acquis aux valeurs du libéralisme économique, le nouveau gouvernement va être désigné. Nous devons exprimer fortement ce que nous attendons de lui [...] Donner de l'oxygène aux entreprises [...] des réformes de fond [...]. Les 5 chemins de la compétitivité : désendetter la nation; financer l'économie par l'épargne; emploi : flexibilité du temps de travail et des rémunérations; équilibre des systèmes sociaux; partage des responsabilités entre le système éducatif et le monde de l'entreprise... (AG 1992)

\section{Les occurrences du terme social}

Quatre lexies dominent le champ social : protection sociale (de base avec 198 occurrences) et sécurité sociale (73 occurrences avec un suremploi Medef), partenaires sociaux (203 occurrences) et dialogue social (114 occurrences), en suremploi Medef.

Se réapproprier le champ social. Pourquoi négocier avec les syndicats? Je crois d'abord que la réappropriation du champ social est la meilleure manière d'influer positivement sur la législation, et d'obtenir la souplesse nécessaire à la gestion car je n'ai pas confiance dans la capacité des pouvoirs publics à bâtir par la loi les conditions optimales pour les entrepreneurs. (AG 1995)

Attesté dans le corpus dès 1981, l'emploi de partenaires sociaux (pics d'emploi en 1992, 2001 et 2002) et de dialogue social (pics d'emploi en 1998, 2001 et 2002) s'intensifie avec la refondation sociale :

- autres lexies : dépenses sociales, relations sociales, cohésion sociale, justice sociale, progrès social, droit social, domaine social, régimes sociaux, vie économique et sociale, fracture sociale, innovation sociale, négociation sociale : «La flexibilité sur les salaires liée au mérite individuel est une forme de justice sociale, [...] alors que l'égalitarisme n'est que l'expression vertueuse de la jalousie sociale» (AG 1985) :

- lexies majoritairement CNPF : charges sociales, politique sociale, traitement social, rapports sociaux, CSG;

- lexies majoritairement Medef : action sociale, action sanitaire et sociale, démocratie sociale, dette sociale, nouvelle demande sociale, délibération 
sociale, ascension sociale, Europe sociale, France sociale, modernisation sociale, refondation sociale.

Les questions sociales, appréhendées sous l'angle de la compétitivité de l'économie, sont l'objet des réformes. Le social doit servir l'économique, l'économique dicte sa loi au social.

Cette toute première exploration du corpus CNPF/Medef, limitée à l'examen des formes les plus fréquentes et/ou les plus spécifiques, a permis de dessiner l'architecture lexicométrique du discours par laquelle s'expose la doctrine patronale. La maquette qui en ressort figure une construction discursive à trois étages : un premier, en surplomb, positionne les paradigmes centraux du discours nous et entreprise, entités soudées au point de pouvoir se confondre; un second pose les valeurs fondatrices du dogme économique libéral avec les nombreuses formes de base ou peu fluctuantes; un troisième ouvre le dispositif doctrinal aux nécessités de ressourcement pour soutenir dans le temps la crédibilité de l'ensemble. Alors que la radicalisation du discours du président Seillière et la médiatisation de thématiques plus libérales depuis 1997 laissaient attendre une évolution lexicale notable, l'analyse renvoie au contraire une grande stabilité du vocabulaire majeur. Le cœur de l'architecture lexicométrique résiste à toutes les actualisations de l'air du temps jusqu'à frôler «l'ultra-répétition ». Cette ultra-répétition remplit la double fonction d'asseoir la longue durée de la représentation et de disséminer un vocabulaire aussi transparent que l'air que l'on respire. En effet, plus les mots sont fréquents donc répétés - la fréquence n'étant rien d'autre que la répétition -, moins nous y prêtons attention. À l'instar des mots-outils grammaticaux, ils colonisent notre système de pensée : ils pensent pour nous. «On mémorise mieux ce qui se répète. Un système répétitif comme le discours politique, dans ses sloganisations et dans ses attirances d'attirances, est proche de la révélation de la propagande. C'est ce qui peut court-circuiter la rationalisation de l'écoute. La propagande est faite pour l'inattention, comme la publicité » (Tournier, 1998, p. 526). Ce faisant, ils définissent un registre de lieux communs reproduits comme une évidence.

Le corpus a révélé que l'opération lexicale (prévue de longue date et toujours reportée pour ménager la fragile unité interne) qui s’était opérée entre le CNPF et le Medef affectait principalement la terminologie identitaire du groupe avec l'effacement des mots renvoyant à la «classe patronale» et au vocabulaire organisationnel, obstacle à la propagation d'une doctrine en mouvement pour tisser un projet global de société depuis longtemps pressenti. La «douce violence » subie en interne a ainsi dégagé les voies de l'expansion externe, comme l'ont montré les significations amplifiées des mots étudiés. Ainsi, le nous du Medef, c'est la France, ce sont les entreprises, non pas les employeurs mais l'unité économique enrôlant au passage les salariés : signe d'un véritable 
changement de paradigme dans lequel disparaît la référence au patronat pour promouvoir une sorte d'intérêt général de la société dont le slogan, «l'entreprise, c'est la vie », donne un raccourci saisissant.

\section{Références}

Amossé Thomas, Flocco Gaëtan, Lefèvre Josette, Pernot Jean-Marie, Petit Héloïse, Rey Frédéric, TALLard Michèle, Tuchszirer Carole, Vincent Catherine, 2012, Les organisations patronales. Continuités et mutations des formes de représentation du patronat, Rapport de recherche, Paris, IRES-CEE.

BERGOUNIOUX Alain, LAUNAY Michel F. et al., 1982, La parole syndicale, Paris, PUF.

BÉRoud Sophie, LefÈVRE Josette, 2010, «Le corpus syndical. Une expérience au long cours », Mots. Les langages du politique, n094, novembre, p. 97-106.

BoltANSKI Luc, ChiAPEllo Ève, 1999, Le nouvel esprit du capitalisme, Paris, Gallimard. Bourdieu Pierre, Boltanskı Luc, 1976, «La production de l'idéologie dominante», Actes de la recherche en sciences sociales, vol. II, n²-3, juin.

BUnEL Jean, 1996, "Les dilemmes de l'action patronale», La Revue de l'IRES, n²0, p. 5-34.

CoulouARn Tanguy, 2008, «Au nom des patrons. L'espace de représentation patronale en France ", thèse de doctorat en science politique sous la dir. de M. Offerlé, Université Paris 1.

Dudouet François-Xavier, GRÉMONT Éric, 2010, Les grands patrons en France. Du capitalisme d'État à la financiarisation, Paris, Lignes de repères.

Fraboulet Danièle, 2007, Quand les patrons s'organisent. Stratégies et pratiques de l'Union des industries métallurgiques et minières, 1901-1950, Lille, Presse universitaire du Septentrion.

Fraboulet Danièle, Druelle-Kom Clotilde, Vernus Pierre éd., 2013, Les organisations patronales et la sphère publique. Europe, $\mathrm{xIX}^{\mathrm{e}}$ et $\mathrm{xx}$ siècles, Rennes, PUR.

GARRIC Nathalie, LÉGLISE Isabelle, 2008, «Le discours patronal, un exemple de discours économique?», Mots. Les langages du politique, n 86, p. 67-83.

GUILBERT Thierry, 2008, Le discours idéologique ou la Force de l'évidence, Paris, L'Harmattan.

HARRIS André, SÉdouy Alain (de), 1977, Les patrons, Paris, Le Seuil.

Hetzel Anne-Marie, LefÈvre Josette, Mouriaux René, Tournier Maurice, 1998, Le syndicalisme à mots découverts. Dictionnaire des fréquences (1971-1990), Paris, Syllepse.

Kolboom Ingo, 1984, «Patron et patronat. Histoire sociale du concept de patronat en France au $19^{\mathrm{e}}$ et $20^{\mathrm{e}}$ siècle », Mots, nº 9 , p. 89-112.

- 1986, La revanche des patrons. Le patronat français face au Front populaire, Paris, Flammarion.

LATOU Rn ERIE Gilles, 1996, La chronologie du patronat français, Paris, Cirnov.

LEBARON Frédéric, 2000, La croyance économique. Les économistes entre science et politique, Paris, Le Seuil. 
LefÈVRE Josette, 1993, «CFDT 1973-1992 : l'éclatement d'un champ lexical», Mots. Les langages du politique, $\mathrm{n}^{\circ} 36$, septembre, p. 85-100.

- 2003, «De l'univers lexical de travailleurs à celui de salariés. La CFDT de 1970 à 1998 : évolution d'un parcours identitaire», Constructions identitaires et dynamiques politiques, L. Baugnet éd., Bruxelles, Peter Lang, p. 193-211.

MAGNIADAS Jean, 2002, Un héritier du capitalisme dynastique, Montreuil, Vo Éditions.

Maingueneau Dominique, Cossuta Frédéric, 1995, "L'analyse des discours constituants », Langages, nº117, p. 112-125.

Michel Hélène éd., 2013, Représenter le patronat européen. Formes d'organisation patronale et modes d'action européenne, Bruxelles, Peter Lang.

Mots, mars 1981, n²2, Qu'est-ce que le vocabulaire spécifique d'un texte politique?

Mots, mars 1985, nº10, Le nous politique.

Mouriaux René, Narritsens André éd., 2012, Lexique usuel critique de l'idéologie dominante économique et sociale, Montreuil, Institut CGT d'histoire sociale.

OfFERLÉ Michel, 2007, «Les organisations patronales », Problèmes politiques et sociaux, $\mathrm{n}^{\circ} 937$.

- 2009, Sociologie des organisations patronales, Paris, La Découverte.

- 2013, Les patrons des patrons. Histoire du Medef, Paris, Odile Jacob.

Tournier Maurice, 1998, Mélanges Maurice Tournier. Des mots en liberté, Lyon, ENS Éditions.

WeBer Henri, 1986, Le parti des patrons. Le CNPF1946-1990, Paris, Le Seuil. 